\title{
Expression of epithelial membrane and 3-fucosyl-N- acetyllactosamine antigens in cervix uteri with particular reference to adenocarcinoma in situ
}

\author{
T P ROLLASON,* P BYRNE, $\dagger$ ANN WILliAMS $\ddagger$ G BROWN
}

From the *Department of Pathology, University of Birmingham and Department of Histology, The Birmingham and Midland Hospital for Women; the $†$ Department of Obstetrics and Gynaecology. The Birmingham and Midland Hospital for Women; the $\ddagger$ Department of Histology, Birmingham Maternity Hospital; and the $\S$ Department of Immunology, University of Birmingham

SUMMARY The staining patterns obtained with antiepithelial membrane antigen (anti-EMA) and the monoclonal antibody to 3-fucosyl-N-acetyllactosamine (AGF 4:48) in the uterine cervix in intraepithelial and invasive neoplasia were compared to determine a possible role in differential diagnosis of reactive and neoplastic conditions. Both early invasive and in situ adenocarcinoma stained equally intensely with both agents and both antibodies stained diffusely tubal metaplasia, endometrial lined glands, and even occasional areas of normal endocervical mucosa.

It is concluded that these agents are unlikely to be of use in the routine histological differentiation of glandular and squamous cervical dysplasia or neoplasia, but immunostaining with anti-EMA may help differentiate between reactive and metaplastic changes in endocervical glands and adenocarcinoma in situ.

Previous studies by other workers, ${ }^{1}$ using a monoclonal antibody related to Dako-EMA (anti-human milk fat globulin 1), have suggested that differentiation between atypical - that is, dysplastic - and typical endocervical glands may be possible on the basis of the pattern of staining achieved because dysplastic glandular epithelium stains diffusely and more intensely.

The carbohydrate group believed to be detected by AGF 4:48 is widely distributed in human tissue. ${ }^{2}$ An association has recently been shown between certain differentiation antigens and neoplastic markers using monoclonal antibodies against carbohydrate determinants of the above type, and previous studies of human breast tissue have indicated that clinically important changes in fucose containing glycoconjugates occur in malignant disease. ${ }^{34}$

It has also been shown that in formalin fixed normal endocervical tissue considerable changes in staining with AGF 4:48 may be achieved by prior treatment with neuraminidase, which may be due to antigen unmasking by removal of neuraminic acid residues.

We compared the staining patterns obtained with antiepithelial membrane antigen (anti-EMA) and AGF 4:48 in the uterine cervix in intraepithelial and

Accepted for publication 5 January 1988 invasive neoplasia to determine whether these monoclonal antibodies could be used in the differential diagnosis of reactive and neoplastic conditions, with particular reference to intraepithelial glandular neoplasia.

\section{Material and methods}

Anti-EMA (Dako-EMA, Dako Ltd, High Wycombe, Buckinghamshire) is a monoclonal antibody raised against a preparation of human milk fat globule membrane protein. ${ }^{56}$ The determinant recognised by this antibody is thought to be carbohydrate in nature. AGF 4:48 is a monoclonal antibody which has been shown to detect the carbohydrate group 3-fucosyl-Nacetyllactosamine ${ }^{2}$ also detected by the monoclonal antibodies VEPF, VEP9, My-1, SSEA1, and IG10 ${ }^{7-9}$

All of the tissues used were fixed in Bouin's solution. All sections were dewaxed and endogenous peroxidase blocked with $0.5 \%$ hydrogen peroxide in methanol for 30 minutes. After rinses in distilled water and phosphate buffered saline (PBS) at pH 7.5, containing $1 \%$ normal swine serum, sections for AGF 4:48 staining were incubated with hybridoma culture supernate, diluted 1 in 3 with PBS, in a damp chamber for one and a half hours. Sections for staining with anti-EMA were 
rinsed in distilled water and left in distilled water for 10 minutes at $37^{\circ} \mathrm{C}$ before immersing in $0.1 \%$ trypsin (Difco) in $0.1 \%$ calcium chloride for 20 minutes at $37^{\circ} \mathrm{C}$. They were then washed in running tap water for 15 minutes, twice in PBS with $1 \%$ normal swine serum for five minutes and then incubated with anti-EMA, diluted 1 in 50 with PBS, in a damp chamber for one hour.

For both antibodies, treatment from this stage was identical and as follows: sections were washed twice in PBS with $1 \%$ normal swine serum, incubated with rabbit antimouse immunoglobulins (Dako, diluted 1 in 40 with PBS) for 30 minutes, washed twice in PBS, incubated with swine antirabbit immunoglobulin (diluted 1 in 40 with PBS) for 30 minutes, washed twice in PBS with $1 \%$ normal swine serum, incubated with PAP (Dako, diluted 1 in 40 with PBS) for 30 minutes, washed twice in PBS with $1 \%$ normal swine serum followed by a PBS wash and incubated with diamino benzidine-hydrogen peroxide solution in PBS for eight minutes. After rinsing in distilled water sections were counterstained with Mayer's haematoxylin for one and a half minutes, blued in tap water, dehydrated, cleared and mounted.

Prior treatment with neuraminidase was performed before blocking endogenous peroxidase by incubating sections rinsed with acetate buffer at $\mathrm{pH} 5.5$ with a solution of one unit per $\mathrm{ml}$ neuraminidase (Type V, Sigma Chemical Co Ltd, Poole, Dorset) and 1\% calcium chloride for one hour and 16 hours at $37^{\circ} \mathrm{C}$, followed by washing with tap water. Trypsinisation before staining with AGF 4:48 was performed as described for EMA staining. Control sections were incubated in buffer.

Sections used were all from cone biopsy specimens and were chosen to permit internal controls on each section-for example, cervical intraepithelial neoplasia, grade III (CIN III), adenocarcinoma in situ and immature metaplasia in one section. Twenty one cases were studied including six cases of adenocarcinoma in situ.

\section{Results}

NORMAL CER VIX

Both anti-EMA and AGF 4:48 stained the ectocervical squamous mucosa variably within and between cases. Occasional areas showed intense staining usually with relative sparing of the suprabasilar zone, but more commonly staining was weak or absent.

Both antisera stained the squamous side of the squamo-columnar junction more intensely than the rest of the squamous mucosa, and this seemed to be a consistent finding.

The normal endocervical surface and glandular mucosa typically stained only on its free (luminal)

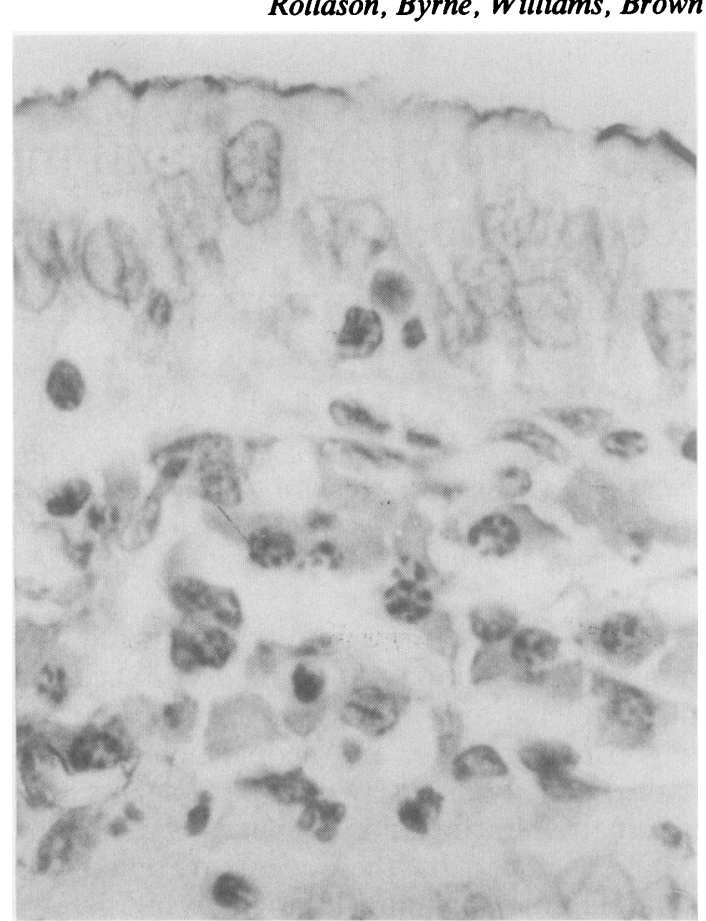

Fig 1 Normal endocervical mucosa showing typical surface staining seen with anti-EMA and (less consistently) with AGF 4:48. (Immunoperoxidase stain, anti-EMA.)

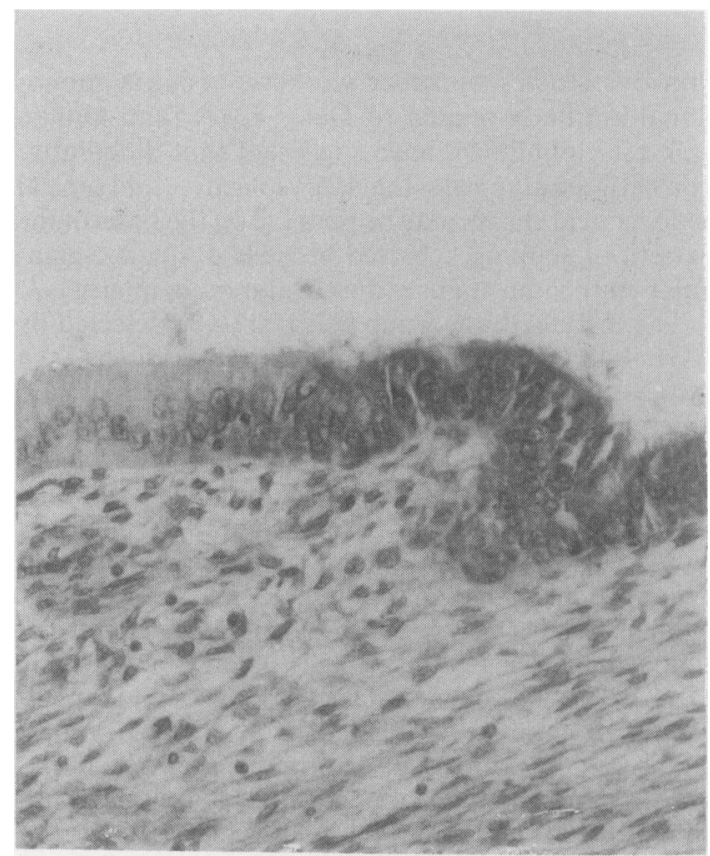

Fig 2 Junction of tubal metaplasia (to right of picture) and normal endocervical mucosa showing AGF 4:48 staining of metaplastic zone. 


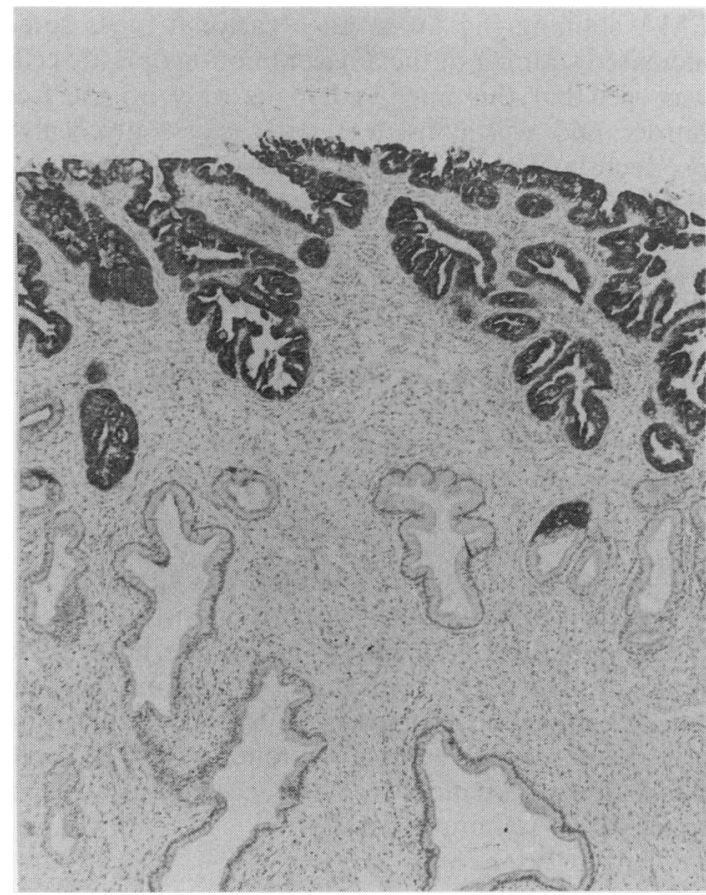

Fig 3 Intense staining of endocervical glands affected by adenocarcinoma in situ but deeper healthy glands are not stained. (Immunoperoxidase stain, anti-EMA.)

surface to give a "pencilled" margin to the cells (fig 1). This was more consistently and intensely seen with anti-EMA but with both antibodies occasional foci of diffuse, usually weak cellular staining were seen in clearly non-dysplastic cells.

\section{NON-NEOPLASTIC CERVICAL CONDITIONS}

Immature metaplastic squamous epithelium and areas of reserve cell hyperplasia generally stained positively with both anti-EMA and AGF 4:48. This staining was not as intense as that of adenocarcinoma in situ but did approach the intensity of staining of some areas of CIN III. Clearly this finding makes it unlikely that either agent would be of use in the cytological differentiation of these cellular changes from neoplasia. Microglandular hyperplasia did not stain with either antibody.

Glands in the endocervix lined by endometrial or endosalpingeal-type epithelium both stained positively with anti-EMA and AGF 4:48. With antiEMA this staining was weak but diffuse, the intensity made it generally easy to distinguish from adenocarcinoma in situ. In the case of AGF 4:48 (fig 2), the intensity of staining was similar to that seen in the more weakly stained areas of adenocarcinoma in situ. Again this clearly limits the usefulness of this antibody

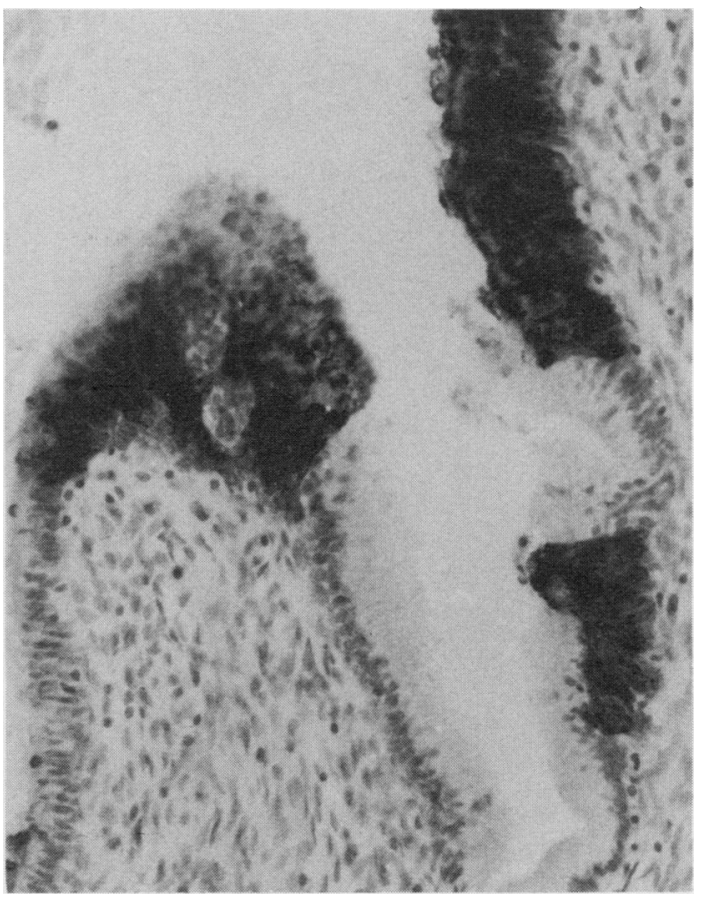

Fig 4 Clearly defined margin is seen between heavily stained adenocarcinoma in situ and normal endocervical mucosa. (Immunoperoxidase stain, anti-EMA.)

in differentiating these conditions, which can occasionally be a source of diagnostic difficulty.

Areas of reactive hyperchromasia in glandular epithelium and squamous epithelium did not stain positively with either antibody.

\section{CER VICAL INTRAEPITHELIAL NEOPLASIA (SQUAMOUS)}

All cases of CIN III studied stained positively with anti-EMA, though staining was variable and patchy. Gland cleft tissue stained similarly to surface mucosa. Findings with AGF 4:48 were similar but more variable; some areas of CIN III stained intensely and others unstained even in the same section.

\section{ADENOCARCINOMA IN SITU}

Anti-EMA stained areas of adenocarcinoma in situ (both gland cleft and surface epithelial) intensely and consistently (fig 3 ). In areas where a mixed pattern of mucin secreting cells and squamous cells was seen staining was equally intense and well defined. Unlike the normal glandular cells, the neoplastic cells stained diffusely throughout the cytoplasm, though some surface accentuation was seen in a few cases. In almost all areas the clear cut division between neoplastic and non-neoplastic glandular cells was reflected in the anti- 


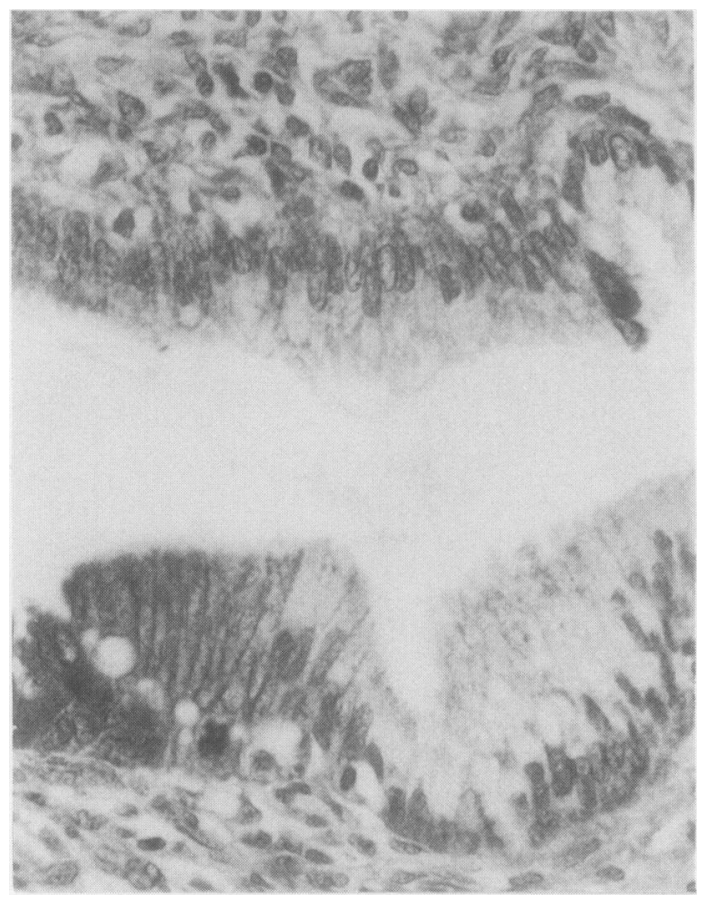

Fig 5 Junction of adenocarcinoma in situ and non-dysplastic endocervical mucosa. (Immunoperoxidase stain, AGF 4:48.)

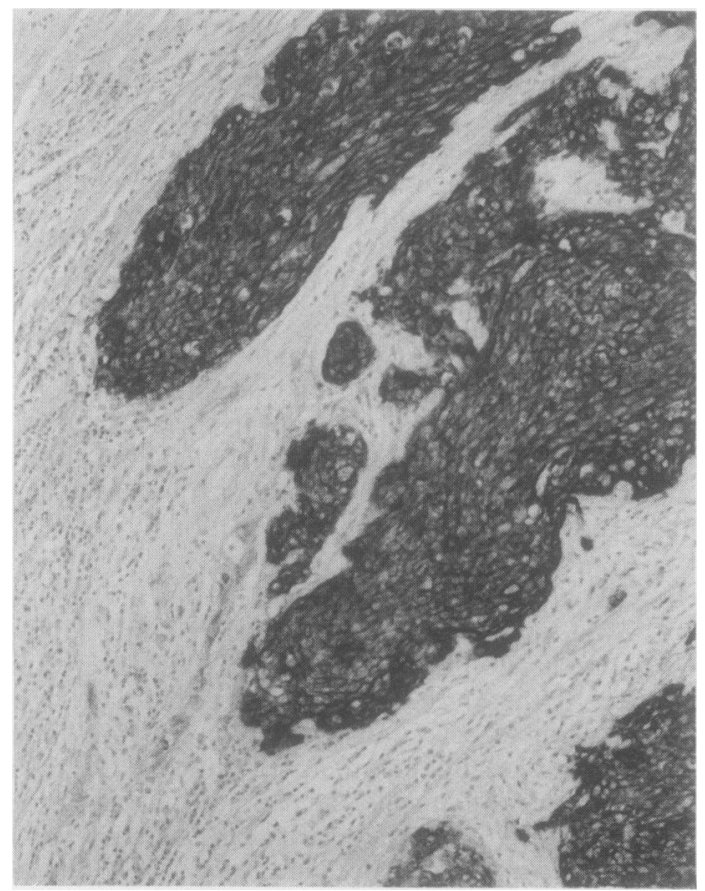

Fig 6 Invasive squamous carcinoma of cervix showing very dramatic staining with anti-EMA.
EMA staining (fig 4). In an occasional focus some increased staining of the adjacent non-neoplastic cells was seen but this appeared to be only on the free border and was consistent with excess glycocalyx production by the adjacent neoplastic cells.

AGF 4:48 stained adenocarcinoma in situ in a very similar manner to anti-EMA (fig 5), but the staining was generally less intense. Occasional areas of adenocarcinoma in situ did not stain at all.

INVASIVE SQUAMOUS, ADENOSQUAMOUS, AND ADENOCARCINOMA

All three disease states stained intensely with antiEMA (fig 6), and positively, though more variably with AGF 4:48.

\section{EFFECT OF NEURAMINIDASE AND PRETREATMENT} WITH TRYPSIN ON AGF 4:48 STAINING

Neuraminidase treatment for either one hour or 16 hours produced no definite increase in staining intensity or change in the staining pattern. Trypsinisation also produced no increase in staining. In fact, both pretreatments seemed, in some cases, to lead to a reduction in staining generally.

\section{Discussion}

Adenocarcinoma in situ of the endocervix was first described over 30 years ago and until recently was considered to be relatively rare, though 160 cases had been reported up until $1984 .{ }^{10}$ Clearly, in the diagnosis of this condition the most important prognostic feature must be the presence or absence of invasion. This is often extremely difficult to determine and assessment of architectural changes, stromal response, and gland depth may all be problematic. Unfortunately, this study does not offer hope for the use of the antibodies investigated in differentiating early invasive from in situ lesions as both conditions stain strongly with both agents.

As stated, it has been suggested ${ }^{1112}$ that glandular atypia of lesser degrees of severity than adenocarcinoma in situ can be recognised in the endocervix and that a monoclonal antibody raised to human milk fat globulin might be useful as a marker for this atypia. ${ }^{1}$ In our studies neither anti-EMA nor AGF 4:48 clearly defined areas of glandular dysplasia of lesser severity than adenocarcinoma in situ adjacent to this condition, though this conclusion is based on subjective interpretation of what degree of cellular pleomorphism constitutes "atypia". The results cannot be regarded as evidence against the existence of glandular dysplasia which should be expected to occur on an empirical basis, and which, we believe, does exist. What does seem to be clear is that in Bouin's fixative both anti-EMA and AGF 4:48 may be used preferen- 
tially (but not specifically) to stain adenocarcinoma in situ. Anti-EMA, particularly, may be of use in the occasional case in differentiating this condition from inflammatory reactive conditions of the glandular cervical epithelium and tubal and endometrial lined glands. The fact that foci of weaker but diffuse staining with both antibodies were seen in tubal metaplasia, endometrial lined glands, and even in a few areas of entirely normal endocervical mucosa makes it highly unlikely that the antibodies could be used alone to differentiate lesser degrees of glandular dysplasia (if they do occur) from benign conditions, unless their staining intensity was similar to that of adenocarcinoma in situ. The positive staining of squamous metaplasia and reserve cell hyperplasia also severely limit the usefulness of anti-EMA and AGF 4:48 in a more general sense.

The different pattern of staining in the cervix with patchy staining before treatment with neuraminidase and staining of all endocervical epithelial cells and mucin after neuraminidase treatment ${ }^{2}$ pointed to the possibility that in malignant or dysplastic conditions "unmasking" of 3-fucosyl- $\mathrm{N}$-acetyllactosamine might occur, leading to increased AGF 4:48 staining. Whether this is the case is not clear but our findings with neuraminidase treatment of normal epithelium are at variance with those previously described. This discrepancy may be due to the difference in the fixative used, but recently a similar absence of an increase in staining after treatment with neuraminidase has been seen by other workers in breast tumours ${ }^{7}$ that had been fixed in formol saline.

This study confirms the findings of Fray et al on staining of normal and in situ neoplastic squamous mucosa and again highlights the difficulty in using anti-EMA (and AGF 4:48) to differentiate neoplastic from non-neoplastic epithelium due to the staining of immature metaplasia and reserve cell hyperplasia. ${ }^{13} \mathrm{~A}$ further problem we have seen in this regard is the increased intensity of staining of the transformation zone.

Our findings also confirm their finding of staining of invasive squamous carcinoma of the cervix by antiEMA. Similar staining was seen with AGF 4:48. By analogy with anti-EMA, AGF 4:48 may also stain tumours at other sites despite the fact that the normal parent epithelium such as squamous mucosa, stains patchily and inconsistently. This possibility clearly needs further investigation.

It has been suggested that a considerable proportion of the invasive carcinomas of the cervix occurring in younger women may be adenosquamous rather than purely squamous or glandular. ${ }^{14}$ Interestingly, there seems to be a mixed pattern of in situ squamous and glandular neoplastic cells seen in conjunction with adenocarcinoma in situ and CIN III which stains intensely and consistently (more so than CIN III) with both anti-EMA and AGF 4:48. This observation offers tentative support for the theory ${ }^{15}$ that both CIN and adenocarcinoma in situ arise from a common reserve cell.

The lack of specificity with regard to anti-EMA staining of epithelial tumours clearly indicates that this antibody cannot be used to differentiate between primary and metastatic adenocarcinoma in the female genital tract. ${ }^{13}$ Preliminary studies of our own indicate that this is also the case for AGF 4:48 as a considerable proportion of ovarian carcinomata also stain positively with this antibody.

In conclusion, our findings with monoclonal antibody to EMA generally confirm those of other authors and indicate that this antibody may have a useful role in the definition of the extent of adenocarcinoma in situ of the endocervix and in the occasional case to differentiate adenocarcinoma in situ from benign metaplasias. We have not been able to identify clearly areas of lesser dysplasia ("atypia") in glandular endocervical cells adjacent to adenocarcinoma in situ, however, with this monoclonal antibody to EMA (clone 29). In the case of human cervix uteri AGF 4:48 seems to give a staining pattern similar to that obtained with anti-EMA. In general, the staining is weaker and less consistent, and AGF 4:48 is unlikely to be of diagnostic use in the cervix.

Our grateful thanks go to Mrs S Smith for her excellent secretarial assistance and to Mr Alan Cooper for photographic help.

\section{References}

1 Brown LJR, Griffin NR, Wells M. Cytoplasmic reactivity with the monoclonal antibody HMFG1 as a marker of cervical glandular atypia. J Pathol 1987;151:203-8.

2 Howie AJ, Brown G. Effects of neuraminidase on the expression of the 3-fucosyl-N-acetyllactosamine antigen in human tissues. $J$ Clin Pathol 1985;38:409-16.

3 Feizi T. Demonstration by monoclonal antibodies that carbohydrate structures of glycoproteins and glycolipids are oncodevelopmental antigens. Nature 1985;314:53-7.

4 Walker RA. The binding of peroxidase-labelled lectins to human breast epithelium. III-Altered fucose-binding patterns of breast carcinomas and their significance. $J$ Pathol 1984;144:109-17.

5 Heyderman E, Strudley I, Powell G, Richardson TC, Cordell JL, Mason DY. A new monoclonal antibody to epithelial membrane antigen (EMA)-E29. A comparison of its immunocytochemical reactivity with polyclonal anti-EMA antibodies and with another monoclonal antibody, HMFG2. Br J Cancer 1985;52:355-61.

6 Cordell J, Richardson TC, Pulford KAF, et al. Production of monoclonal antibodies against human epithelial membrane antigen for use in diagnostic immunocytochemistry. $\mathrm{Br} \mathrm{J}$ Cancer 1985;52:347-54.

7 Walker RA, Day SJ. The expression of fucosylated Type 2 blood group chains in human breast and their significance. $J$ Pathol 1986;149:113-20. 
8 Gooi HC, Feizi T, Kapadia A, Knowles BB, Solter D, Evans MJ. Stage specific embryonic antigen involves 1-3 fucosylated type 2 blood group chains. Nature 1981;292:156-8.

9 Gooi HC, Thorpe SJ, Hunsell EF, Rumbold H, Kraft D, Forster O, Feizi T. Marker of peripheral blood granulocytes and monocytes of man recognised by two monoclonal antibodies VEP8 and VEP9 involves the trisaccharide 3-fucosylN-acetyllactosamine. Eur J Immunol 1983;13:306-12.

10 Ostor AG, Pagano R, Davoren RA, Fortune DW, Charen W, Rome R. Adenocarcinoma-in-situ of the cervix. Int J Gynecol Pathol 1984;3:179-90.

11 Brown LJR, Wells M. Cervical glandular atypia associated with squamous intraepithelial neoplasia: a premalignant lesion? $J$ Clin Pathol 1986;39:22-8.

12 Van Roon E, Boon ME, Kurver PJH, Baak JPA. The association between precancerous columnar and squamous lesions of the cervix: a morphometric study. Histopathology 1983;7:887-96.

13 Fray RE, Husain OAN, To ACW, et al. The value of immunocytochemical markers in the diagnosis of cervical neoplasia. $B J$ Obstet Gynecol 1984;91:1037-41.

14 Gallup DG, Harper RH, Stock RJ. Poor prognosis in patients with adenosquamous cell carcinoma of the cervix. Obstet Gynecol 1985;65:416-22.

15 Boon ME, Kirk RS, Rietveld-Scheffers PEM. The morphogenesis of adenocarcinoma of the cervix-a complex pathological entity. Histopathology 1981;5:565-77.

Requests for reprints to: Dr T P Rollason, Department of Pathology, The Medical School, Edgbaston, Birmingham B15 2TJ, England. 